TRANSACTIONS OF THE

AMERICAN MATHEMATICAL SOCIETY

Volume 363, Number 9, September 2011, Pages 4505-4525

S 0002-9947(2011)05480-2

Article electronically published on April 19, 2011

\title{
THE CORONA FACTORIZATION PROPERTY AND REFINEMENT MONOIDS
}

\author{
EDUARD ORTEGA, FRANCESC PERERA, AND MIKAEL RØRDAM
}

\begin{abstract}
The Corona Factorization Property of a $\mathrm{C}^{*}$-algebra, originally defined to study extensions of $\mathrm{C}^{*}$-algebras, has turned out to say something important about intrinsic structural properties of the $\mathrm{C}^{*}$-algebra. We show in this paper that a $\sigma$-unital $\mathrm{C}^{*}$-algebra $A$ of real rank zero has the Corona Factorization Property if and only if its monoid $\mathrm{V}(A)$ of Murray-von Neumann equivalence classes of projections in matrix algebras over $A$ has a certain (rather weak) comparability property that we call the Corona Factorization Property (for monoids). We show that a projection in such a $\mathrm{C}^{*}$-algebra is properly infinite if (and only if) a multiple of it is properly infinite.

The latter result is obtained from some more general results that we establish about conical refinement monoids. We show that the set of order units (together with the zero-element) in a conical refinement monoid is again a refinement monoid under the assumption that the monoid satisfies weak divisibility; and if $u$ is an element in a refinement monoid such that $n u$ is properly infinite, then $u$ can be written as a sum $u=s+t$ such that $n s$ and $n t$ are properly infinite.
\end{abstract}

\section{INTRODUCTION}

The Corona Factorization Property was introduced by Elliott and Kucerovsky in 7], and studied further by Kucerovsky and $\mathrm{Ng}$ in [10, as a tool to study extensions of $\mathrm{C}^{*}$-algebras. The salient feature of the Corona Factorization Property is that it ensures that (full) extensions are absorbing.

At the level of $\mathrm{C}^{*}$-algebras, the Corona Factorization Property is easily defined: a $C^{*}$-algebra $A$ has this property if and only if every full projection in the multiplier algebra of the stabilization, $A \otimes \mathcal{K}$, is properly infinite.

The failure of having the Corona Factorization Property seems to be a common feature of several examples of "badly behaved" $\mathrm{C}^{*}$-algebras that have been exhibited over the last 5-10 years. The vague phrase "badly behaved" should perhaps be replaced with "infinite dimensional" in a suitable non-commutative sense. The example by the third named author in [16] of a (simple, nuclear) $\mathrm{C}^{*}$-algebra $A$ which is not stable, but where a matrix algebra over $A$ is stable, does not have the Corona Factorization Property. In fact, the existence of a full, non-properly infinite projection in the multiplier algebra over the stabilization of $A$ is the crucial

Received by the editors April 1, 2009.

2000 Mathematics Subject Classification. Primary 46L35, 06F05; Secondary 46L80.

Key words and phrases. $\mathrm{C}^{*}$-algebras, Corona Factorization Property, real rank zero, conical refinement monoids. 
property in that example. It is also crucial for the example, also by the third named author, in [17. of a simple nuclear $\mathrm{C}^{*}$-algebra that contains both a finite and an infinite projection.

It remains unknown if there is an example of a simple $\mathrm{C}^{*}$-algebra of real rank zero which contains both finite and infinite projections. If not, then every simple $\mathrm{C}^{*}$-algebra of real rank zero is either stably finite or purely infinite. It was observed by Zhang that this dichotomy holds for simple $\mathrm{C}^{*}$-algebras of real rank zero that satisfy the Corona Factorization Property.

Many (classes of) $\mathrm{C}^{*}$-algebras are known to satisfy the Corona Factorization Property. These include purely infinite $\mathrm{C}^{*}$-algebras (simple or not), $\mathcal{Z}$-stable algebras (see [8]), simple $\mathrm{C}^{*}$-algebras with real rank zero, stable rank one and weak unperforation on their $K_{0}$-group (so, in particular, all simple AF-algebras), [10, algebras of the type $C(X) \otimes \mathcal{K}$, where $X$ is a finite dimensional compact Hausdorff metric space, [15, and also all unital $\mathrm{C}^{*}$-algebras with finite decomposition rank, 11.

We show that a $\sigma$-unital $\mathrm{C}^{*}$-algebra $A$ of real rank zero (simple or not) satisfies the Corona Factorization Property if and only if its monoid $\mathrm{V}(A)$ of Murray-von Neumann equivalence classes of projections satisfies a certain (rather weak) comparability property that we call the Corona Factorization Property (for monoids). We also characterize the $\sigma$-unital $\mathrm{C}^{*}$-algebras $A$ of real rank zero where every ideal has the Corona Factorization Property as those where $\mathrm{V}(A)$ satisfies a (more natural) comparability property, which we call the strong Corona Factorization Property (for monoids).

We further show that $\mathrm{C}^{*}$-algebras with the Corona Factorization Property (simple or not) satisfy an analog of the dichotomy for simple $\mathrm{C}^{*}$-algebras of real rank zero with the Corona Factorization Property mentioned above. This is first established at the level of monoids. More precisely, if $M$ is a conical refinement monoid with the strong Corona Factorization Property, and if $u$ is an element in $M$ such that a multiple of $u$ is properly infinite, then $u$ itself is properly infinite. (We say that $u$ is properly infinite if $2 u \leq u$.) If $M$ has the Corona Factorization Property, then the statement above only holds for order units. This result can then be translated into a statement about $\mathrm{C}^{*}$-algebras of real rank zero with the Corona Factorization Property, saying that any full projection $p$ in the stabilization of such a $\mathrm{C}^{*}$-algebra, for which some multiple $p \oplus p \oplus \cdots \oplus p$ is properly infinite, is itself properly infinite.

In outline, the paper is as follows. After recalling basic definitions, we proceed in Section 2 to define the concept of weak divisibility of order units, a property that implies that order units can be split into a sum of several order units. We prove that any conical refinement monoid with a properly infinite order unit has weak divisibility of order units. This includes $\mathrm{V}(A)$, for any properly infinite $\mathrm{C}^{*}$-algebra $A$ with real rank zero.

Section 3 is devoted to showing that in a refinement monoid $M$ with weak divisibility of order units, the submonoid $M^{*} \cup\{0\}$ of order units together with zero is a simple refinement monoid. This allows for the reduction of certain arguments to the simple case. For example, we show that for a real rank zero algebra $A$ such that $\mathrm{V}(A)$ has weak divisibility of order units and strict unperforation, then the subsemigroup of classes of full projections is cancellative. A further consequence is obtained in Section 4, where we show that, in a conical refinement monoid with an 
order unit $u$ such that $n u$ is properly infinite, $u=s+t$ for order units $s$ and $t$ such that $n s$ and $n t$ are also properly infinite.

Finally, in Section 5 we obtain the results, described above, on when $\sigma$-unital $\mathrm{C}^{*}$-algebras of real rank zero have the Corona Factorization Property, and we show that (full) projections in such $\mathrm{C}^{*}$-algebras are properly infinite if some multiples of them are properly infinite.

\section{Preliminaries}

All monoids in this paper will be abelian, written additively. The main object of study in this paper is the monoid of (Murray-von Neumann) equivalence classes of projections associated to a $\mathrm{C}^{*}$-algebra. We briefly recall its construction below.

We say that two projections $p$ and $q$ in a $\mathrm{C}^{*}$-algebra $A$ are Murray-von Neumann equivalent provided there is a partial isometry $v$ such that $p=v v^{*}$ and $v^{*} v=q$. One can extend this to the set $\mathcal{P}\left(M_{\infty}(A)\right)$ of projections in $M_{\infty}(A)$, which becomes a congruence, and thereby construct

$$
\mathrm{V}(A)=\mathcal{P}\left(M_{\infty}(A)\right) / \sim
$$

where $[p] \in \mathrm{V}(A)$ stands for the equivalence class that contains the projection $p$ in $M_{\infty}(A)$. This becomes an abelian monoid with operation $[p]+[q]=[p \oplus q]$, where $p \oplus q$ refers to the matrix $\left(\begin{array}{ll}p & 0 \\ 0 & q\end{array}\right)$.

We say that a monoid $M$ is conical if $x+y=0$ only when $x=y=0$. Notice that, for any $\mathrm{C}^{*}$-algebra $A$, the projection monoid $\mathrm{V}(A)$ is conical. Therefore, we shall assume that all our monoids are conical although, for emphasis, this assumption will be repeated in our statements of results.

A class of $\mathrm{C}^{*}$-algebras for which the monoid $\mathrm{V}(A)$ captures a good deal of their structure is that of real rank zero. Recall that $A$ has real rank zero provided that the set of self-adjoint, invertible elements is dense in the set of all self-adjoint elements (see [5]). It is well known that this condition allows us to produce projections on demand, as is equivalent, for example, to the statement asserting that each hereditary subalgebra contains an approximate unit consisting of projections.

As shown by Ara and Pardo ([1]), based on work of Zhang, for $\mathrm{C}^{*}$-algebras with real rank zero, the projection monoid enjoys the additional property of having refinement (see, e.g., 6], 18), which we now proceed to define:

We say that a monoid $M$ is a refinement monoid if whenever $a_{1}+a_{2}=b_{1}+b_{2}$ in $M$, there exist elements $z_{i j}$ in $M$ such that $a_{i}=z_{1 i}+z_{2 i}$ and $b_{i}=z_{i 1}+z_{i 2}$ for $i=1,2$. The elements $z_{i j}$ are referred to as a refinement of the original equation. For ease of notation, we shall write these equations as follows:

\begin{tabular}{c|cc} 
& $a_{1}$ & $a_{2}$ \\
\hline$b_{1}$ & $z_{11}$ & $z_{12}$ \\
$b_{2}$ & $z_{21}$ & $z_{22}$
\end{tabular}

and refer to the above display as a refinement matrix.

All abelian monoids have a natural pre-order, the algebraic ordering, defined as follows: if $x, y \in M$, we write $x \leq y$ if there is a $z$ in $M$ such that $x+z=$ $y$. In the case of $\mathrm{V}(A)$, the algebraic ordering is given by Murray-von Neumann subequivalence, that is, $[p] \leq[q]$ if and only if there is a projection $p^{\prime} \leq q$ such that $p \sim p^{\prime}$. We also write, as is customary, $p \precsim q$ to mean that $p$ is subequivalent to $q$. 


\section{WEAK DIVISIBILITY}

The purpose of this section is to consider a weak form of divisibility that appears quite frequently both in ring theory and operator algebras (see [14 and [3]). In the sequel it will be important to apply this to the set of full projections, which in the monoid-theoretical context corresponds to the set of order units.

Let us recall that an element $u$ in a monoid $M$ is an order unit provided $u \neq 0$ and, for any $x$ in $M$, there is $n \in \mathbb{N}$ such that $x \leq n u$. In the case that every non-zero element of $M$ is an order unit, then $M$ is said to be simple. Recall that a projection $p$ of a $\mathrm{C}^{*}$-algebra $A$ is full if the closed, two-sided ideal it generates is $A$. This is equivalent to saying that $[p]$ is an order unit as an element of $\mathrm{V}(A)$. If $A$ is a simple $\mathrm{C}^{*}$-algebra, then $\mathrm{V}(A)$ is a simple monoid.

For a monoid $M$, let us denote by $M^{*}$ the subsemigroup of order units of $M$ (so in the simple case, $\left.M^{*}=M \backslash\{0\}\right)$. Notice that $M^{*} \cup\{0\}$ is a submonoid of $M$.

Lemma 2.1. Let $M$ be a conical refinement monoid. Given two order units $u$ and $v$ in $M$, there exists an order unit $w \in M$ such that $w \leq u, v$.

Proof. Since $u$ is an order unit, there exist $n \in \mathbb{N}$ and $t \in M$ such that $v+t=n u$. It follows then from [18, Lemma 1.9] that there is a refinement

\begin{tabular}{c|cccc} 
& $u$ & $u$ & $\cdots$ & $u$ \\
\hline$v$ & $v_{1}$ & $v_{2}$ & $\cdots$ & $v_{n}$ \\
$t$ & $t_{1}$ & $t_{2}$ & $\cdots$ & $t_{n}$
\end{tabular}

with $v_{1} \leq v_{2} \leq \cdots \leq v_{n}$ and $t_{1} \geq t_{2} \geq \cdots \geq t_{n}$. Set $w=v_{n}$, which is an order unit since $v$ is an order unit and $v \leq n w$.

Definition 2.2. Let $M$ be a monoid. An element $x$ in $M$ will be termed weakly divisible if there exist $a$ and $b$ in $M$ such that $x=2 a+3 b$. We say that $M$ is weakly divisible if every element is weakly divisible. We say that $M$ has weak divisibility for order units if every order unit is weakly divisible.

Weak divisibility of order units immediately enables us to decompose order units into sums of order units, as the following lemma testifies:

Lemma 2.3. Let $M$ be a monoid that has weak divisibility of order units. Given any order unit $u \in M$ there exist order units $v, w \in M$ such that $u=v+w$ and $v \leq w$.

Proof. By assumption, there are elements $x$ and $y$ in $M$ with $u=2 x+3 y$. Put $v=x+y$ and $w=x+2 y$. Then $v$ and $w$ are order units since $u \leq 3 v$ and $u \leq 2 w$. Clearly $u=v+w$ and $v \leq w$.

Notation 2.4. For elements $x, y$ in a monoid $M$, we will use $x \leq^{*} y$ to mean $x+z=y$ for an order unit $z$ in $M$. If $M$ is simple, then all we are asking for is that $z \neq 0$. In particular, in the simple case, $x<y$ (taken to mean $x \leq y$ and $x \neq y$ ) implies $x \leq^{*} y$.

Our observation above yields:

Corollary 2.5. Let $M$ be a conical refinement monoid with weak divisibility of order units. Given two order units $u, v$ in $M$, there is an order unit $w$ such that $w \leq * u, v$. 
Proof. According to Lemma 2.3 we may write $u=u_{1}+u_{2}$ and $v=v_{1}+v_{2}$, where $u_{i}$ and $v_{i}$ are order units. Now apply Lemma 2.1 to find an order unit $w$ such that $w \leq u_{2}, v_{2}$, whence $w \leq^{*} u, v$.

Recall that an ideal of a monoid $M$ is a submonoid $I$ which is hereditary with respect to the algebraic ordering. In other words, for $x$ and $y$ in $M$, we have $x+y \in I$ if and only if $x, y \in I$. For a $\mathrm{C}^{*}$-algebra $A$ and a closed, two-sided ideal $I$ of $A$, the monoid $\mathrm{V}(I)$ naturally becomes an ideal of $\mathrm{V}(A)$. Once we have an ideal, we can define a congruence on $M$ by declaring $x \sim y$ if there are elements $a, b$ in $I$ such that $x+a=y+b$. Then $M / I=M / \sim$ naturally becomes a monoid, whose elements will be denoted by $\bar{x}$, for $x$ in $M$. In this language, simple monoids are those which do not have non-trivial ideals.

If $M$ is a refinement monoid and $I$ is an ideal of $M$, then it is easy to see that both $I$ and $M / I$ are refinement monoids (see [2]). Again, for $\mathrm{C}^{*}$-algebras of real rank zero, the natural quotient map induces an isomorphism $\mathrm{V}(A) / \mathrm{V}(I) \cong \mathrm{V}(A / I)$ (see [2]).

Recall that a non-zero element $x$ in a monoid $M$ is an atom (or irreducible) if whenever $x=a+b$, then either $a=0$ or $b=0$. A monoid without atoms is called atomless.

Given an element $u$ in a monoid $M$, let us denote by $\langle u\rangle$ the submonoid generated by $u$, that is, $\langle u\rangle=\{0, u, 2 u, \ldots\}$. Observe that if $M$ is a refinement monoid, then $u$ is an atom in $M$ if and only if $\langle u\rangle$ is an ideal of $M$ and $\langle u\rangle$ is isomorphic to $\mathbb{Z}^{+}$ (via $u \mapsto 1$ ). It was shown in [3, Theorem 6.7] (see also Remark 2.8 below) that an element in a conical refinement monoid is weakly divisible if and only if it is not an atom in any quotient. Combining this result with the observation made above, we get the following:

Proposition 2.6. Let $M$ be a conical refinement monoid. Then $M$ is weakly divisible if and only if no non-zero quotient of $M$ has an ideal isomorphic to $\mathbb{Z}^{+}$.

As an immediate consequence, we obtain the following corollary:

Corollary 2.7. A conical simple refinement monoid $M$ is atomless, and hence weakly divisible, if and only if it is not isomorphic to $\mathbb{Z}^{+}$.

Remark 2.8. In the countable case, which will be of interest as $\mathrm{V}(A)$ is countable whenever $A$ is a separable $\mathrm{C}^{*}$-algebra, Proposition 2.6 can be obtained by the arguments in [14. We briefly indicate how to proceed in that case. First, recall that a dimension monoid is, by definition, an inductive limit of simplicial monoids or, equivalently, a monoid that can be represented as $\mathrm{V}(A)$ for an AF-algebra $A$. Now, if $M$ is a countable refinement monoid, use [14, Theorem 3.9] to find a (countable) dimension monoid $\Delta$ and a surjective map $\alpha: \Delta \rightarrow M$ such that $x \propto y$ if and only if $\alpha(x) \propto \alpha(y)$. (Here, $x \propto y$ means that $x \leq n y$ for some natural number $n$.) In particular, such a map induces an isomorphism between the ideal lattices of $\Delta$ and $M$. By the observation above, that $\langle u\rangle$ is an ideal in $M$ and $\langle u\rangle \cong \mathbb{Z}^{+}$if and only if $u$ is an atom in $M$, one can see that the map $\alpha$ also induces a one-to-one correspondence between atoms in $\Delta$ and atoms in $M$. This allows us to reduce the problem to dimension monoids, and in that case the result holds after [14, Proposition 5.6].

Recall that a non-zero element $x$ in a monoid $M$ is termed infinite provided that there is a non-zero element $y$ such that $x=x+y$. The (non-zero) element $x$ is 
properly infinite if $2 x \leq x$ (whence $m x \leq x$ for all $m \in \mathbb{N}$ ). It follows from the definitions that, if $x \leq y$ and $x$ is infinite, then so is $y$. If $x$ is properly infinite and $x \leq y$, then $y$ need not be properly infinite. However, if we also assume that $y \propto x$, then $y$ is necessarily properly infinite. (Indeed, if $x \leq y \leq n x$ and $x$ is properly infinite, then $2 y \leq 2 n x \leq x \leq y$.)

Evidently, every properly infinite element is infinite. It is well known that the converse also holds in the simple case. Indeed, if $M$ is simple and $x \in M$ is infinite, then $x=x+y$ for some non-zero $y$ in $M$. Hence $x=x+m y$ for all $m \in \mathbb{N}$. Now, $y$ is an order unit, whence there is some $m \in \mathbb{N}$ such that $2 x \leq m y \leq x$, and so $x$ is properly infinite.

Recall that a (non-zero) projection $p$ in a $\mathrm{C}^{*}$-algebra is properly infinite if $p \oplus p \precsim$ $p$, which is of course equivalent to the element $[p] \in \mathrm{V}(A)$ being properly infinite as defined above.

We record the following easy facts for future reference:

Lemma 2.9. Let $M$ be a conical monoid with a properly infinite order unit u. Then:

(i) There is a properly infinite order unit $v$ with $u \leq v$ and $v+u=u$.

(ii) For any other order unit $w$, there is $n \in \mathbb{N}$ such that $n w$ is properly infinite.

Proof. (i). Since $2 u \leq u$, there is an element $t$ such that $2 u+t=u$. Now put $v=u+t$.

(ii). Given an order unit $w$, there is $n \in \mathbb{N}$ with $u \leq n w$. Since $u$ is properly infinite, $n w \leq u$. Now, $2 n w \leq u+u \leq u \leq n w$.

Observe that, if $M$ is an abelian monoid, $I$ is an ideal in $M$, and $u \in I$, then $u$ is infinite (respectively, properly infinite) in $M$ if and only if $u$ is infinite (respectively, properly infinite) in $I$. Also, if $u \notin I$ and $u$ is properly infinite, then $\bar{u}$ is properly infinite in $M / I$.

Proposition 2.10. Let $M$ be a conical refinement monoid with a properly infinite order unit. Then $M$ has weak divisibility for order units.

Proof. Let $u$ be a properly infinite order unit in $M$, and let $v$ be any other order unit. By Lemma 2.9, there is $n \in \mathbb{N}$ such that $n v$ is properly infinite.

If $v$ is not weakly divisible, then, by [3, Theorem 6.7], $\bar{v}$ is an atom in some quotient $M / I$ of $M$ (so in particular $v \notin I$ ). Since $\langle\bar{v}\rangle$ is isomorphic to $\mathbb{Z}^{+}$, we see that $\bar{v}$ and $n \bar{v}$ are necessarily finite. But this contradicts the fact that $n v$ is properly infinite in $M$ and hence also in $M / I$.

Corollary 2.11. Let $A$ be a properly infinite $C^{*}$-algebra with real rank zero. Then $\mathrm{V}(A)$ has weak divisibility of order units.

\section{REFinement OF ORDER Units}

The property of refinement in an abelian monoid is preserved under the passage to ideals, as is well known and easy to show. However, refinement will often be lost when considering submonoids of the original monoid. One of our aims in this section is to show that, under the presence of weak divisibility, the subsemigroup $M^{*}$ of order units of a refinement monoid $M$ will also have the refinement property. From this, it follows that $M^{*} \cup\{0\}$ is a simple refinement monoid. This will allow us to reduce the proof of some of our results to the simple case. The lemma below is due to Ken Goodearl. 
Lemma 3.1. Let $M$ be a conical refinement monoid and suppose that we are given a refinement

\begin{tabular}{c|cc} 
& $c_{1}$ & $c_{2}$ \\
\hline$a$ & $a_{1}$ & $a_{2}$ \\
$b$ & $b_{1}$ & $b_{2}$
\end{tabular}

in $M$, such that $c_{1} \geq c_{2}$. Then there exists a refinement

\begin{tabular}{c|cc} 
& $c_{1}$ & $c_{2}$ \\
\hline$a$ & $a_{1}^{\prime}$ & $a_{2}^{\prime}$ \\
$b$ & $b_{1}^{\prime}$ & $b_{2}^{\prime}$
\end{tabular}

such that $a_{1}^{\prime} \geq a_{2}^{\prime}$. If $b_{2}$ is an order unit, the refinement above can be taken so that furthermore $b_{2}^{\prime}$ is an order unit.

Proof. We have $c_{1}=c_{2}+x$ for some $x$, whence $a_{1}+b_{1}=a_{2}+\left(b_{2}+x\right)$. The latter equation has a refinement

\begin{tabular}{c|cc} 
& $a_{2}$ & $b_{2}+x$ \\
\hline$a_{1}$ & $y_{11}$ & $y_{12}$ \\
$b_{1}$ & $y_{21}$ & $y_{22}$.
\end{tabular}

Now set $a_{1}^{\prime}=y_{11}+y_{12}+y_{21}$ and $a_{2}^{\prime}=y_{11}$, while $b_{1}^{\prime}=y_{22}$ and $b_{2}^{\prime}=b_{2}+y_{21}$. Then

\begin{tabular}{c|cc} 
& $c_{1}$ & $c_{2}$ \\
\hline$a$ & $a_{1}^{\prime}$ & $a_{2}^{\prime}$ \\
$b$ & $b_{1}^{\prime}$ & $b_{2}^{\prime}$
\end{tabular}

is a refinement with $a_{1}^{\prime} \geq a_{2}^{\prime}$. Moreover $b_{2}^{\prime} \geq b_{2}$, so that $b_{2}^{\prime}$ is an order unit if $b_{2}$ is.

Recall that $M^{*}$ stands for the subsemigroup of order units of a conical abelian monoid, and that $x \leq^{*} y$ means that $x+z=y$ for some $z$ in $M^{*}$.

Lemma 3.2. Let $M$ be a conical refinement monoid with weak divisibility of order units. Suppose we have a refinement

\begin{tabular}{c|cc} 
& $c$ & $d$ \\
\hline$a$ & $x_{11}$ & $x_{12}$ \\
$b$ & $x_{21}$ & $x_{22}$
\end{tabular}

in $M$, where $x_{11}, x_{22} \in M^{*}$ (hence also $a, b, c, d \in M^{*}$ ). Then, there exists a refinement

\begin{tabular}{c|cc} 
& $c$ & $d$ \\
\hline$a$ & $z_{11}$ & $z_{12}$ \\
$b$ & $z_{21}$ & $z_{22}$
\end{tabular}

with $z_{i j}$ in $M^{*}$ for all $i, j$.

Proof. By Corollary 2.5. there is an order unit $u$ such that $u \leq^{*} x_{11}, x_{22}$, so we may write $x_{11}=u+z_{11}$ and $x_{22}=u+z_{22}$, where both $z_{11}$ and $z_{22}$ belong to $M^{*}$. Now define $z_{12}=u+x_{12}$ and $z_{21}=u+x_{21}$. It is now a simple matter to check that $z_{i j}$ are order units that give the refinement of the conclusion. 
Lemma 3.3. Let $M$ be a conical refinement monoid with weak divisibility of order units. If we have

$$
a+b=c+d,
$$

where $a, b, c, d \in M^{*}$, there is then a refinement matrix

\begin{tabular}{c|cc} 
& $c$ & $d$ \\
\hline$a$ & $z_{11}$ & $z_{12}$ \\
$b$ & $z_{21}$ & $z_{22}$
\end{tabular}

with $z_{11} \in M^{*}$.

Proof. Use Lemma 2.3 to split $a=x+y$ and $c=u+v$, where $u, v, x, y \in M^{*}$ and $v \leq u, y \leq x$. By Lemma 3.1, there is a refinement

\begin{tabular}{c|cc} 
& $v$ & $u+d$ \\
\hline$y$ & $x_{11}$ & $x_{12}$ \\
$b+x$ & $x_{21}$ & $x_{22}$
\end{tabular}

with $x_{11} \leq x_{21}$, so in particular $x_{21}$ is an order unit. A second application of Lemma 3.1 yields a refinement

\begin{tabular}{c|cc} 
& $v$ & $u+d$ \\
\hline$y$ & $x_{11}^{\prime}$ & $x_{12}^{\prime}$ \\
$b+x$ & $x_{21}^{\prime}$ & $x_{22}^{\prime}$,
\end{tabular}

where $x_{21}^{\prime} \in M^{*}$ and $x_{11}^{\prime} \leq x_{12}^{\prime}$, so in particular $x_{12}^{\prime} \in M^{*}$. We may thus apply Lemma 3.2 and assume at the outset that the elements $x_{i j}$ are all order units.

Refine the equality $b+x=x_{21}+x_{22}$ and get a refinement matrix

$$
\begin{array}{c|cc} 
& x_{21} & x_{22} \\
\hline x & a_{11} & a_{12} \\
b & a_{21} & a_{22} .
\end{array}
$$

Putting $z_{11}^{\prime}=x_{11}+a_{11}, z_{12}^{\prime}=x_{12}+a_{12}$, we obtain a refinement

$$
\begin{array}{c|cc} 
& v & u+d \\
\hline a & z_{11}^{\prime} & z_{12}^{\prime} \\
b & a_{21} & a_{22},
\end{array}
$$

where $z_{11}^{\prime}, z_{12}^{\prime} \in M^{*}$. Proceeding in the same way for the equality $u+d=z_{12}^{\prime}+a_{22}$, we obtain the desired result.

Of course, we can arrange, in Lemma 3.3. any of the entries of the final refinement to be an order unit. Arranging them all to be order units simultaneously is somewhat more delicate, and we deal with this in the result below.

Theorem 3.4. Let $M$ be a conical refinement monoid with weak divisibility of order units. If we have

$$
a+b=c+d,
$$

where $a, b, c, d \in M^{*}$, there is then a refinement matrix

\begin{tabular}{c|cc} 
& $c$ & $d$ \\
\hline$a$ & $z_{11}$ & $z_{12}$ \\
$b$ & $z_{21}$ & $z_{22}$
\end{tabular}

with $z_{i j} \in M^{*}$ for all $i, j$. In particular, $M^{*} \cup\{0\}$ is a simple refinement monoid. 
Proof. Retain the notation and procedure in the proof of the previous lemma up to the refinement

\begin{tabular}{c|cc} 
& $v$ & $u+d$ \\
\hline$a$ & $z_{11}^{\prime}$ & $z_{12}^{\prime}$ \\
$b$ & $a_{21}$ & $a_{22}$,
\end{tabular}

where $z_{11}^{\prime}$ and $z_{12}^{\prime}$ are both order units.

Since $v \leq u$, we may apply Lemma 3.1 to obtain a refinement matrix

$$
\begin{array}{c|cc} 
& v & u+d \\
\hline a & t_{11} & t_{12} \\
b & t_{21} & t_{22},
\end{array}
$$

where $t_{11} \in M^{*}$ and $t_{21} \leq t_{22}$, so in particular also $t_{22} \in M^{*}$. Applying Lemma 3.2 if necessary, we may assume then that all the $t_{i j}$ 's are order units, by probably losing the inequality $t_{21} \leq t_{22}$.

Next, refine $u+d=t_{12}+t_{22}$ applying Lemma 3.3 to obtain

\begin{tabular}{c|cc} 
& $u$ & $d$ \\
\hline$t_{12}$ & $w_{11}$ & $w_{12}$ \\
$t_{22}$ & $w_{21}$ & $w_{22}$,
\end{tabular}

with $w_{22} \in M^{*}$. Finally, put $z_{11}=t_{11}+w_{11}, z_{12}=w_{12}, z_{21}=t_{21}+w_{21}$ and $z_{22}=w_{22}$, where at least $z_{11}$ and $z_{22}$ are order units. A final application of Lemma 3.2 yields the desired result.

Remark 3.5. In the simple atomless case, our result above says that a non-zero refinement problem $a+b=c+d$ (with $a, b, c, d$ being non-zero) admits a non-zero refinement matrix (i.e., with all entries being non-zero), a result which is well known and much easier to prove.

Corollary 3.6. Let $M$ be a conical refinement monoid with weak divisibility of order units. If $a, b, c \in M^{*}, n \geq 1$, and $a+b=n c$, there are order units $u_{1}, \ldots, u_{n}$, $v_{1}, \ldots, v_{n}$ and $z_{1}, \ldots, z_{n-1}$ such that $u_{i}=u_{i+1}+z_{i}$ and $v_{i}+z_{i}=v_{i+1}$ for every $i=1, \ldots, n-1$, and such that

\begin{tabular}{c|cccc} 
& $u$ & $u$ & $\cdots$ & $u$ \\
\hline$n u$ & $u_{1}$ & $u_{2}$ & $\cdots$ & $u_{n}$ \\
$v$ & $v_{1}$ & $v_{2}$ & $\cdots$ & $v_{n}$
\end{tabular}

is a refinement matrix.

Proof. By Theorem 3.4 $M^{*} \cup\{0\}$ is a simple refinement monoid, whence Lemma 1.9 in [18] and its proof can be applied to obtain a refinement in $M^{*}$ (that is, in $M^{*} \cup\{0\}$ with all elements being non-zero) such as the one in our statement.

We now draw another consequence of Theorem 3.4 .

Definitions 3.7. We remind the reader that a monoid $M$ is almost unperforated if whenever $(k+1) x \leq k y$ for $k \in \mathbb{N}$, it follows that $x \leq y$. More generally, $M$ has $n$ comparison if whenever $x, y_{0}, y_{2}, \ldots, y_{n}$ are elements in $M$ such that $x<_{s} y_{j}$ for all $j=0,1, \ldots, n$, then $x \leq y_{0}+y_{1}+\cdots+y_{n}$. Here $x<_{s} y$ means that $(k+1) x \leq k y$ for some natural number $k$. It follows immediately from the definitions that $M$ is almost unperforated if and only if $M$ has 0 -comparison. Notice that if $M$ has $n$-comparison, then $M$ has $m$-comparison for any $m \geq n$. 
Recall also that a monoid $M$ is termed strictly unperforated if, whenever $n x+z=$ $n y$ for $x, y, z \in M$ with $z \neq 0$ and $n \in \mathbb{N}$, there is a non-zero element $w$ such that $x+w=y$.

That these properties are equivalent for conical simple refinement monoids is quite possibly well known. We state the result and outline the proof for the sake of completeness.

Proposition 3.8. Let $M$ be a simple conical refinement monoid with order unit $u$. Then, the following conditions are equivalent:

(i) $M$ is almost unperforated.

(ii) $M$ has n-comparison for any $n$.

(iii) $M$ has $n$-comparison for some $n$.

(iv) $M$ is strictly unperforated.

(v) For every non-zero element $x$ in $M$ such that $x \leq u$, there exists $k \in \mathbb{N}$ such that, if $y \in M$ and $k y \leq u$, then also $y \leq x$.

Proof. If $M$ is atomic, then it is isomorphic to the infinite cyclic monoid, by [1, Lemma 1.6], and all five conditions are easily seen to hold in that case. We may thus assume that $M$ is non-atomic, and in particular it will be atomless. This implies that $M$ is weakly divisible (see Corollary 2.7), so it has weak divisibility for order units.

It is clear that (i) $\Rightarrow$ (ii) $\Rightarrow$ (iii), and also that (iv) $\Rightarrow$ (i). That (iv) and (v) are equivalent follows from [13, Lemma 3.7].

Let us check, finally, that (iii) $\Rightarrow(\mathrm{v})$. Assume $M$ has $n$-comparison for some $n$. Let $x \in M$ be non-zero and assume $x \leq u$. Since $M$ is weakly divisible, we may write $x=x_{0}+\cdots+x_{n}$ with all $x_{i}$ non-zero (hence all order units). There is $k^{\prime} \in \mathbb{N}$ such that $u \leq k^{\prime} x_{i}$ for all $i$. Now let $k=k^{\prime}+1$. If $y \in M$ and $k y \leq u$, then $\left(k^{\prime}+1\right) y \leq k^{\prime} x_{i}$ for all $i$, whence $n$-comparison implies that $y \leq x_{0}+\cdots+x_{n}=x$.

Condition (v) above was termed weak comparability (see [1]).

Lemma 3.9 (cf. [12, Lemma 5.1 (a)]). Let $M$ be a conical refinement monoid with weak divisibility of order units. Given order units $x_{1}, \ldots, x_{k} \in M$ and $n \in \mathbb{N}$, there exists an order unit $y \in M$ such that $n y \leq{ }^{*} x_{i}$ for all $i$.

Proof. This follows by applying Theorem 3.4 which implies that $M^{*} \cup\{0\}$ is a simple refinement monoid, and then using condition (a) of Lemma 5.1 in [12.

Corollary 3.10. Let $M$ be a conical refinement monoid with weak divisibility of order units. If $M^{*} \cup\{0\}$ is strictly unperforated, then $M^{*}$ is a cancellative monoid. This holds in particular if $M$ is strictly unperforated.

Proof. By Theorem [3.4, we have that $M^{*} \cup\{0\}$ is a simple, conical, refinement monoid. We may then use Proposition [3.8, together with [1, Theorem 1.7], to conclude that $M^{*}$ is cancellative.

Let us now check that if $M$ is strictly unperforated, then so is $M^{*} \cup\{0\}$. Suppose that $n x+z=n y$, for $x, y \in M^{*} \cup\{0\}$ and $z \in M^{*}$ (so clearly $y \neq 0$ ). If $x=0$, then $0+y=y$.

If $x \in M^{*}$, then using Lemma 3.9. find an order unit $w$ such that $n w \leq^{*} z$. This implies then that $n(x+w)+z^{\prime}=n y$, for some non-zero element $z^{\prime}$; hence strict unperforation in $M$ implies $x+w+w^{\prime}=y$, for some non-zero element $w^{\prime}$. Since $w+w^{\prime}$ is an order unit, we see that $M^{*} \cup\{0\}$ is strictly unperforated. 
Corollary 3.11. Let $A$ be a $C^{*}$-algebra with real rank zero. If $\mathrm{V}(A)$ has weak divisibility for order units and is strictly unperforated, then the subsemigroup of equivalence classes of full projections is cancellative.

We close by developing divisibility results for not necessarily simple conical refinement monoids with weak divisibility of order units. In the simple atomless case (where weak divisibility is automatic by Corollary 2.7), these were obtained in [12, although there only Riesz decomposition was assumed. We remark that our results below hold true in this more general context, but we shall not need this.

Theorem 3.12 (cf. [12, Theorem 5.2]). Let $M$ be a conical refinement monoid with weak divisibility of order units. If $p$ and $r$ are order units, and $m \in \mathbb{N}$, then there are order units $q$ and $s$ with $s \leq r, q$, and $p=m q+s$.

Proof. By Theorem 3.4, $M^{*} \cup\{0\}$ is a simple refinement monoid, so [12, Theorem 5.2] applies.

The proof of the following result is identical to the one given in the simple case. Simplicity there is only assumed to ensure that the element $r$ in the statement below is an order unit.

Lemma 3.13 (cf. [12, Lemma 5.1 (b)]). Let $M$ be a conical refinement monoid, and let $p, r \in M$ with $r$ an order unit. Given $m \in \mathbb{N}$, there exist $q, s \in M$ such that $p=m q+s$ with $s \leq(m-1) r$.

Proposition 3.14. Let $M$ be a conical refinement monoid with weak divisibility of order units. Let $p, r \in M$, with $r$ an order unit, and let $m \in \mathbb{N}$. Then $p=m q+s$ for some $q$ and $s$ in $M$ such that $s \leq r$.

Proof. By Lemma 3.9. there exists an order unit $r^{\prime} \in M$ such that $(m-1) r^{\prime}<r$. Now apply Lemma 3.13 to $p$ and $r^{\prime}$. Then there exist $q$ and $s$ in $M$ such that $p=m q+s$ with $s \leq(m-1) r^{\prime}<r$, and the result follows.

\section{Properly infinite order Units}

In this section we prove a decomposition result for properly infinite order units in a refinement monoid that will be important for our applications to the Corona Factorization Property for $\mathrm{C}^{*}$-algebras discussed in the next section. We will greatly benefit from the results in the previous section, which allow us to reduce to the simple case. We begin with a technical lemma:

Lemma 4.1. Let $M$ be a conical simple refinement monoid. Suppose we are given non-zero elements $s, t, a_{1}, a_{2}, b_{1}, b_{2}, z$ in $M$ and $n \in \mathbb{N}$ such that:

(i) $n s+a_{1}+a_{2}$ and $n t+b_{1}+b_{2}$ are infinite elements.

(ii) $a_{1}+z=s$ and $t+z=b_{1}$.

Then, there are elements $s^{\prime}, t^{\prime}, p, q$ in $M$ such that

$$
s^{\prime}+t^{\prime}=s+t, \quad s^{\prime}=a_{1}+p, \quad t^{\prime}=t+q, \quad z=p+q,
$$

and such that the two elements $(n+1) s^{\prime}+a_{2}$ and $(n+1) t^{\prime}+b_{2}$ are infinite.

Proof. Put $b=n t+b_{1}+b_{2}$, which by assumption is an infinite element (hence properly infinite). We may thus write $b=b+c$ with $c$ an infinite element. There 
is a refinement

\begin{tabular}{c|ccccc} 
& $t$ & $\cdots$ & $t$ & $b_{1}$ & $b_{2}$ \\
\hline$b$ & $d_{1}$ & $\cdots$ & $d_{n}$ & $d_{n+1}$ & $d_{n+2}$ \\
$c$ & $e_{1}$ & $\cdots$ & $e_{n}$ & $e_{n+1}$ & $e_{n+2}$,
\end{tabular}

where all entries are non-zero, except possibly $d_{n+2}$ and $e_{n+2}$.

Use [12, Theorem 5.2] to find elements $x, y$ in $M$ such that $z=(n+1) x+y$, with $y \leq e_{n}$. Set $p=n x+y, q=x$, and

$$
s^{\prime}=a_{1}+n x+y=a_{1}+p, \quad t^{\prime}=x+t=t+q .
$$

Then $p+q=z$ and $s^{\prime}+t^{\prime}=a_{1}+t+z=s+t$. Further,

$$
\begin{aligned}
(n+1) s^{\prime}+a_{2} & =(n+1) a_{1}+(n+1) n x+(n+1) y+a_{2} \\
& \geq(n+1) a_{1}+n(n+1) x+n y+a_{2} \\
& =(n+1) a_{1}+n z+a_{2}=n s+a_{1}+a_{2},
\end{aligned}
$$

so that $(n+1) s^{\prime}+a_{2}$ is infinite. Also,

$$
\begin{aligned}
(n+1) t^{\prime}+b_{2} & =(n+1) t+(n+1) x+b_{2} \\
& \geq d_{1}+\cdots+d_{n-1}+d_{n}+e_{n}+t+(n+1) x+b_{2} \\
& \geq d_{1}+\cdots+d_{n-1}+d_{n}+y+t+(n+1) x+b_{2} \\
& =d_{1}+\cdots+d_{n}+t+z+b_{2} \\
& \geq d_{1}+\cdots+d_{n+2}=b,
\end{aligned}
$$

whence $(n+1) t^{\prime}+b_{2}$ is infinite, as desired.

Proposition 4.2. Let $M$ be a simple, conical refinement monoid, and let $u$ be a non-zero element of $M$. Suppose that nu is infinite for some $n \in \mathbb{N}$. Then there exist $s, t \in M$ with $u=s+t$ such that ns and nt are infinite.

Proof. Write $n u=n u+v$, where $v \in M$ is infinite. Then using [18, Lemma 1.9] and its proof (see also Corollary 3.6) we can find non-zero elements $u_{1} \geq \cdots \geq u_{n}$ and $v_{1} \leq \cdots \leq v_{n}$ with non-zero complements $u_{i}=u_{i+1}+z_{i}$ and $v_{i}+z_{i}=v_{i+1}$ for every $i=1, \ldots, n-1$ such that

\begin{tabular}{c|cccc} 
& $u$ & $u$ & $\cdots$ & $u$ \\
\hline$n u$ & $u_{1}$ & $u_{2}$ & $\cdots$ & $u_{n}$ \\
$v$ & $v_{1}$ & $v_{2}$ & $\cdots$ & $v_{n}$
\end{tabular}

is a refinement matrix.

We show by induction that for each $k=1,2, \ldots, n$ there are elements $s_{k}$ and $t_{k}$ in $M$ such that $s_{k}+t_{k}=u$, and the two elements

$$
k s_{k}+\left(u_{k+1}+\cdots+u_{n}\right), \quad k t_{k}+\left(v_{k+1}+\cdots+v_{n}\right)
$$

are infinite. Moreover, if $k<n$, then there is an element $z_{k}^{\prime}$ such that $u_{k+1}+z_{k}^{\prime}=s_{k}$ and $t_{k}+z_{k}^{\prime}=v_{k+1}$. For $k=n$ the two elements displayed above are equal to $n s_{n}$ and $n t_{n}$, respectively, and so it will follow that $s=s_{n}$ and $t=t_{n}$ have the desired properties.

For $k=1$ we can take $s_{1}=u_{1}, t_{1}=v_{1}$, and $z_{1}^{\prime}=z_{1}$. Assume that $1 \leq k<n$ and that $s_{k}, t_{k}$, and $z_{k}^{\prime}$ have been found. Then it follows from Lemma 4.1 that there are elements $s_{k+1}, t_{k+1}, p, q \in M$ such that

$$
s_{k+1}+t_{k+1}=s_{k}+t_{k}=u, \quad s_{k+1}=u_{k+1}+p, \quad t_{k+1}=t_{k}+q, \quad p+q=z_{k}^{\prime},
$$


and such that $(k+1) s_{k+1}+\left(u_{k+2}+\cdots+u_{n}\right)$ and $(k+1) t_{k+1}+\left(v_{k+2}+\cdots+v_{n}\right)$ are infinite. If $k<n-1$, then put $z_{k+1}^{\prime}=z_{k+1}+p$ and calculate:

$$
\begin{aligned}
u_{k+2}+z_{k+1}^{\prime} & =u_{k+2}+z_{k+1}+p=u_{k+1}+p=s_{k+1}, \\
t_{k+1}+z_{k+1}^{\prime} & =t_{k}+q+z_{k+1}+p=t_{k}+z_{k}^{\prime}+z_{k+1}=v_{k+1}+z_{k+1}=v_{k+2} .
\end{aligned}
$$

Theorem 4.3. Let $M$ be a conical refinement monoid. Let $u$ be an order unit such that nu is properly infinite for some $n \in \mathbb{N}$. Then there exist order units $s, t \in M$ with $u=s+t$, and such that ns and nt are properly infinite.

Proof. We first note that $M$ has weak divisibility of order units, by Proposition 2.10. Thus Theorem 3.4 applies to conclude that $M^{*} \cup\{0\}$ is a simple refinement monoid. Since $n u$ is properly infinite (hence infinite as an element of $M^{*} \cup\{0\}$ ), we may use Proposition 4.2 to find elements $s$ and $t$ in $M^{*}$ with $u=s+t$, and such that $n s$ and $n t$ are properly infinite in $M^{*} \cup\{0\}$, and so also in $M$.

Corollary 4.4. Let $M$ be a conical refinement monoid. If $u$ is an order unit and $n u$ is properly infinite for some $n \in \mathbb{N}$, then there is a sequence $t_{1}, t_{2}, t_{3}, \ldots$ of order units in $M$ such that

$$
t_{1}+t_{2}+\cdots+t_{k} \leq u
$$

for all $k$, and $n t_{i}$ is properly infinite for all $i$.

Proof. By Theorem 4.3, there are order units $s_{1}$ and $t_{1}$ such that $u=s_{1}+t_{1}$ and $n s_{1}$ and $n t_{1}$ are properly infinite. Continuing inductively, we may split each $s_{i}=s_{i+1}+t_{i+1}$ for some order units $s_{i+1}, t_{i+1}$ such that $n s_{i+1}$ and $n t_{i+1}$ are properly infinite.

\section{The Corona Factorization Property in monoids}

Recall that a $\mathrm{C}^{*}$-algebra $A$ is said to have the Corona Factorization Property (CFP) if and only if every full projection in $\mathcal{M}(A \otimes \mathcal{K})$, the multiplier algebra of $A \otimes \mathcal{K}$, is properly infinite. In this section we shall translate the CFP into a comparability property of the $\mathrm{C}^{*}$-algebra $A$ itself (rather than its multiplier algebra) under the assumption that $A$ is of real rank zero. We begin by phrasing this comparability property at the level of monoids.

Definition 5.1. A conical monoid $M$ is said to have the strong Corona Factorization Property (strong CFP) if whenever $x, y_{1}, y_{2}, \ldots$ are elements in $M$ and $m$ is a natural number, one has

$$
\forall n \in \mathbb{N}: x \leq m y_{n} \Longrightarrow \exists k \in \mathbb{N}: x \leq y_{1}+y_{2}+\cdots+y_{k} .
$$

The property of monoids that matches the Corona Factorization Property for $\mathrm{C}^{*}$-algebras of real rank zero is weaker than the property defined above (see Theorem (5.8). The strong CFP considered above is perhaps more natural to study than the weaker one defined below, and it also matches a property of $\mathrm{C}^{*}$-algebras (see Theorem 5.13 below). To define the weaker notion of the Corona Factorization Property we first need the following:

Definition 5.2. A sequence $\left\{x_{n}\right\}_{n=1}^{\infty}$ in a monoid $M$ is said to be full if it is increasing and if for every $y \in M$ there are natural numbers $n$ and $m$ such that $y \leq m x_{n}$. 
Remark 5.3. Every countable monoid $M=\left\{m_{0}, m_{1}, \ldots\right\}$ has a full sequence. Indeed, the sequence $\left\{x_{n}\right\}$ given by $x_{n}:=m_{0}+\cdots+m_{n}$ does the job.

The constant sequence $\left\{x_{n}\right\}$, where $x_{n}=x$ for all $n$, is full if and only if the element $x$ is an order unit.

Full sequences are introduced to make up for the fact that a monoid need not contain an order unit.

Definition 5.4. A conical monoid $M$ is said to satisfy the Corona Factorization Property (CFP) if for every full sequence $\left\{x_{n}\right\}$ in $M$, for every sequence $\left\{y_{n}\right\}$ in $M$, and for every natural number $m$, one has

$$
\forall n \in \mathbb{N}: x_{n} \leq m y_{n} \Longrightarrow \exists k \in \mathbb{N}: x_{1} \leq y_{1}+y_{2}+\cdots+y_{k} .
$$

It is clear that every (conical) monoid satisfying the strong CFP also satisfies the CFP. The two notions clearly agree for simple (conical) monoids.

If $M$ has the CFP and $\left\{x_{n}\right\},\left\{y_{n}\right\}$, and $m$ are as above with $x_{n} \leq m y_{n}$ for all $n$, then for all natural numbers $n$ and $k$ there exists a natural number $l$ such that $x_{n} \leq y_{k}+y_{k+1}+\cdots+y_{l}$. (To see this, one needs only consider the case where $k \geq n$. Next, as $x_{n} \leq x_{k}$, it suffices to consider the case where $n=k$. Now apply the CFP to the sequences $\left\{x_{i}\right\}_{i \geq n}$ and $\left\{y_{i}\right\}_{i \geq n}$.)

Example 5.5. Every almost unperforated conical monoid satisfies the strong CFP. More generally, if $M$ is a conical monoid which has $n$-comparison for some natural number $n$, then $M$ has the strong CFP (see Definition 3.7).

Indeed, suppose that $M$ has $n$-comparison, suppose that $x, y_{1}, y_{2}, \ldots$ are elements in $M$, and $m$ is a natural number such that $x \leq m y_{j}$ for all $j$. Put

$$
z_{j}=y_{j(m+1)+1}+y_{j(m+1)+2}+\cdots+y_{j(m+1)+m+1}, \quad j=0,1, \ldots, n .
$$

Then $(m+1) x \leq m z_{j}$, whence $x<_{s} z_{j}$ for all $j$, which by the definition of $n$ comparison implies that

$$
x \leq z_{0}+z_{1}+\cdots+z_{n}=y_{1}+y_{2}+\cdots+y_{n(m+1)+m+1} .
$$

This shows that $M$ has the strong CFP.

We now relate the $\mathrm{CFP}$ of a monoid to the $\mathrm{CFP}$ of a $\mathrm{C}^{*}$-algebra, and hence we express the CFP for $\mathrm{C}^{*}$-algebras in terms of a comparability property of the $\mathrm{C}^{*}$ algebra. First we need two (well-known) lemmas about comparison of projections in a multiplier algebra:

Lemma 5.6. Let $A$ be a $\sigma$-unital stable $C^{*}$-algebra, let $P$ be a properly infinite, full projection in $\mathcal{M}(A)$, and let $p \leq P$ be a projection in $A$. Then $P-p$ is properly infinite and full in $\mathcal{M}(A)$.

Proof. The assumptions on $P$ and $A$ imply that $P \sim 1$, i.e., that $P=S S^{*}$ for some isometry $S$ in $\mathcal{M}(A)$. Upon replacing $p$ by $S^{*} p S \in A$ we may assume that $P=1$.

Note that $(1-p) A(1-p)$ is $\sigma$-unital because $A$ is. Thus we may apply [9, Corollary 4.3] (and its proof) to conclude that $(1-p) A(1-p)$ is stable. Hence $1-p$, being the unit of the multiplier algebra of the stable $\mathrm{C}^{*}$-algebra $(1-p) A(1-p)$, is properly infinite. Again using that $A$ is stable, by [9, Theorem 3.3] (and its proof), we find that $p \precsim 1-p$. Hence $1 \sim(1-p) \oplus p \precsim(1-p) \oplus(1-p)$, which shows that $1-p$ is full in $\mathcal{M}(A)$. 
Lemma 5.7. Let $A$ be a $C^{*}$-algebra, let $\left\{p_{n}\right\}$ and $\left\{q_{n}\right\}$ be sequences of pairwise orthogonal projections in $A$ such that the sums $P=\sum_{n=1}^{\infty} p_{n}$ and $Q=\sum_{n=1}^{\infty} q_{n}$ are strictly convergent in the multiplier algebra $\mathcal{M}(A)$, and hence define projections $P$ and $Q$ in $\mathcal{M}(A)$.

(i) Suppose that there are sequences $\left\{k_{n}\right\}$ and $\left\{l_{n}\right\}$ of natural numbers such that $1 \leq k_{1}<l_{1}<k_{2}<l_{2}<k_{3}<\cdots$, and such that

$$
\left[p_{n}\right] \leq\left[q_{k_{n}}\right]+\left[q_{k_{n}+1}\right]+\cdots+\left[q_{l_{n}}\right]
$$

for all $n$. Then $P \precsim Q$ in $\mathcal{M}(A)$.

(ii) If $P \precsim Q$ in $\mathcal{M}(A)$, then for every natural number $k$ there exists a natural number $l$ such that

$$
\left[p_{1}\right]+\left[p_{2}\right]+\cdots+\left[p_{k}\right] \leq\left[q_{1}\right]+\left[q_{2}\right]+\cdots+\left[q_{l}\right]
$$

in $\mathrm{V}(A)$.

Proof. (i). For each $n$, let $s_{n} \in A$ be a partial isometry with

$$
s_{n}^{*} s_{n}=p_{n}, \quad s_{n} s_{n}^{*} \leq q_{k_{n}}+q_{k_{n}+1}+\cdots+q_{l_{n}} .
$$

As the sums $\sum p_{n}$ and $\sum q_{n}$ are strictly convergent, it follows that the sum $S=$ $\sum_{n=1}^{\infty} s_{n}$ is strictly convergent in $\mathcal{M}(A)$. Hence $P=S^{*} S \sim S S^{*} \leq Q$.

(ii). Suppose that we are given a partial isometry $S \in \mathcal{M}(A)$ such that $P=S^{*} S$ and $S S^{*} \leq Q$. Put

$$
s_{0}=S\left(p_{1}+p_{2}+\cdots+p_{k}\right) .
$$

Then $s_{0}$ is a partial isometry in $A$ satisfying $s_{0}^{*} s_{0}=p_{1}+p_{2}+\cdots+p_{k}$ and $s_{0} s_{0}^{*} \leq Q$. A standard argument, see e.g. [17, Lemma 4.4], now shows that $p_{1}+p_{2}+\cdots+p_{k} \precsim$ $q_{1}+q_{2}+\cdots+q_{l}$ for some natural number $l$.

Theorem 5.8. Let $A$ be a $C^{*}$-algebra such that $A \otimes \mathcal{K}$ has a countable approximate unit consisting of projections.

(i) If $A$ has the Corona Factorization Property (for $C^{*}$-algebras), then $\mathrm{V}(A)$ has the Corona Factorization Property (for monoids).

(ii) Suppose that $A$ is of real rank zero. Then $A$ has the Corona Factorization Property (for $C^{*}$-algebras) if and only if $\mathrm{V}(A)$ has the Corona Factorization Property (for monoids).

Proof. We may identify $\mathrm{V}(A \otimes \mathcal{K})$ with $\mathrm{V}(A)$, and hence, upon replacing $A$ with $A \otimes \mathcal{K}$, we may assume that $A$ is stable and that $A$ has a countable approximate unit consisting of projections.

(i). Suppose that $\mathrm{V}(A)$ does not satisfy the CFP; i.e., there exist a full sequence $\left\{x_{n}\right\}$ in $\mathrm{V}(A)$, another sequence $\left\{y_{n}\right\}$ in $\mathrm{V}(A)$, and a natural number $m$ such that $x_{n} \leq m y_{n}$ for every $n$, while $x_{1} \nless y_{1}+y_{2}+\cdots+y_{k}$ for every natural number $k$.

Take sequences of pairwise orthogonal projections $\left\{p_{n}\right\}$ and $\left\{q_{n}\right\}$ in $A$ with $\left[q_{n}\right]=x_{n}$ and $\left[p_{n}\right]=y_{n}$ for every $n$, and such that the sums $Q:=\sum_{n=1}^{\infty} q_{n}$ and $P:=\sum_{n=1}^{\infty} p_{n}$ are strictly convergent in $\mathcal{M}(A)$, and hence define projections $Q$ and $P$ in $\mathcal{M}(A)$. We claim that $Q$ is equivalent to $1=1_{\mathcal{M}(A)}$. By the assumption that $A$ has a countable approximate unit consisting of projections, we can write $1=\sum_{n=1}^{\infty} e_{n}$ (with the sum being strictly convergent) for a suitable sequence $\left\{e_{n}\right\}$ of pairwise orthogonal projections in $A$. Since $\left\{x_{n}\right\}$ is a full sequence, there exist natural numbers $m_{j}$ and $k_{j}$ such that

$$
\left[e_{j}\right] \leq m_{j} x_{k_{j}} \leq x_{k_{j}}+x_{k_{j}+1}+\cdots+x_{k_{j}+m_{j}-1} .
$$


Upon replacing each $k_{j}$ with a larger number we can assume that $k_{j+1} \geq k_{j}+m_{j}$. It now follows from Lemma 5.7 (i) that $1 \precsim Q$ in $\mathcal{M}(A)$. As 1 is properly infinite and $K_{0}(\mathcal{M}(A))=0$, it follows that $1 \sim Q$ as claimed.

The $m$-fold direct sum $P \oplus P \oplus \cdots \oplus P$ is equivalent to the projection $\sum_{n=1}^{\infty} p_{n}^{\times}$, where $\left\{p_{n}^{\times}\right\}$is a sequence of pairwise orthogonal projections in $A$ whose sum converges strictly, and where $\left[p_{n}^{\times}\right]=m\left[p_{n}\right]$ for all $n$. As $\left[q_{n}\right]=x_{n} \leq m y_{n}=\left[p_{n}^{\times}\right]$we conclude from Lemma 5.7 (i) that $1 \precsim P \oplus P \oplus \cdots \oplus P$, whence $P$ is full in $\mathcal{M}(A)$.

We finally observe that $Q \npreceq P$ in $\mathcal{M}(A)$. Otherwise, by Lemma 5.7 (ii), we would have $x_{1}=\left[q_{1}\right] \leq\left[p_{1}\right]+\left[p_{2}\right]+\cdots+\left[p_{k}\right]=y_{1}+y_{2}+\cdots+y_{k}$ for some natural number $k$, contradicting the hypothesis.

We have now shown that $P$ is a full projection in $\mathcal{M}(A)$ and that $P$ is not properly infinite (otherwise, $P$ would dominate any other projection in $\mathcal{M}(A)$ ). Hence $A$ does not have the Corona Factorization Property.

(ii). Suppose that $A$ is of real rank zero, and that $\mathrm{V}(A)$ has the CFP. We show that $A$ has the CFP. Take a full projection $P \in \mathcal{M}(A)$, and let $m$ be a natural number such that the $m$-fold direct sum $P \oplus P \oplus \cdots \oplus P$ is properly infinite (and hence equivalent to 1 ). We must show that $P$ itself is properly infinite.

As in (i), write $1=1_{M(A)}=\sum_{n=1}^{\infty} e_{n}$. Since $A$ is $\sigma$-unital and of real rank zero, the hereditary subalgebra $P A P$ has a countable approximate unit consisting of projections. (Indeed, if $\left\{e_{n}\right\}$ is an approximate unit for $A$, then $\left\{P e_{n} P\right\}$ is an approximate unit for $P A P$, whence $P A P$ is $\sigma$-unital, and hence has a countable approximate unit consisting of projections, because it also is of real rank zero.) It follows that we can write $P=\sum_{n=1}^{\infty} p_{n}$ (the sum being strictly convergent), where the $p_{n}$ 's are pairwise orthogonal projections in $P A P$. As in the proof of (i), the $m$-fold direct sum $P \oplus P \oplus \cdots \oplus P$ is equivalent to a projection in $\mathcal{M}(A)$ of the form $\sum_{n=1}^{\infty} p_{n}^{\times}$, where $\left[p_{n}^{\times}\right]=m\left[p_{n}\right]$ for all $n$.

Put $x_{n}=\left[e_{1}\right]+\left[e_{2}\right]+\cdots+\left[e_{n}\right]$. Then $\left\{x_{n}\right\}$ is a full sequence in $\mathrm{V}(A)$. Indeed, let $f$ be an arbitrary projection in $A$. Then $f \precsim 1_{\mathcal{M}(A)}$, whence $[f] \leq x_{n}$ for some $n$ by Lemma 5.7 (ii).

We proceed to show that there is a sequence $1=k_{1}<k_{2}<k_{3}<\cdots$ of natural numbers such that

(1) $x_{n} \leq\left[p_{k_{n}}^{\times}\right]+\left[p_{k_{n}+1}^{\times}\right]+\cdots+\left[p_{k_{n+1}-1}^{\times}\right]=m\left(\left[p_{k_{n}}\right]+\left[p_{k_{n}+1}\right]+\cdots+\left[p_{k_{n+1}-1}\right]\right)$

for all $n$. The existence of $k_{2}$ such that (1) holds for $n=1$ follows from Lemma 5.7 (ii) applied to the relation $1=\sum_{n=1}^{\infty} e_{n} \precsim \sum_{n=1}^{\infty} p_{n}^{\times}$. To establish (11) for $n=2$ use Lemma 5.6 to see that $\sum_{n=k_{2}}^{\infty} p_{n}^{\times}$is properly infinite and full. Applying Lemma 5.7 (ii) to the resulting relation $1=\sum_{n=1}^{\infty} e_{n} \precsim \sum_{n=k_{2}}^{\infty} p_{n}^{\times}$yields $k_{3}>k_{2}$ such that (1) holds for $n=2$. Continue in this manner to find the remaining $k_{j}$ 's.

Put $y_{n}=\left[p_{k_{n}}\right]+\left[p_{k_{n}+1}\right]+\cdots+\left[p_{k_{n+1}-1}\right]$. Then $x_{n} \leq m y_{n}$ for all $n$ by (1). We next claim that there exists a sequence $1=l_{1}<l_{2}<l_{3}<\cdots$ of natural numbers such that

$$
x_{n} \leq y_{l_{n}}+y_{l_{n}+1}+\cdots+y_{l_{n+1}-1}
$$

for all $n$. The existence of $l_{2}$ such that (2) holds for $n=1$ follows directly from the assumption that $\mathrm{V}(A)$ has the CFP. Now apply the CFP to the sequences $\left\{x_{n}\right\}$ and $\left\{y_{n}^{\prime}\right\}$, where $y_{n}^{\prime}=y_{n+l_{1}}$, noting that $x_{n} \leq x_{n+l_{1}} \leq m y_{n+l_{1}}=m y_{n}^{\prime}$. Then we get $l_{3}>l_{2}$ such that (2) holds for $n=2$. Continue in this manner to find the remaining $l_{j}$ 's. 
Put $j_{n}=k_{l_{n}}$. Then, by (2), we have

$$
\left[e_{n}\right] \leq x_{n} \leq\left[p_{j_{n}}\right]+\left[p_{j_{n}}+1\right]+\cdots+\left[p_{j_{n+1}-1}\right]
$$

for all $n$. We can now conclude from Lemma 5.7 (i) that $1 \precsim P$, whence $P$ is properly infinite as desired.

Combine the result above with Example [5.5 to get the following:

Corollary 5.9. Let $A$ be a $\sigma$-unital $C^{*}$-algebra of real rank zero, and suppose that $\mathrm{V}(A)$ is almost unperforated, or that $\mathrm{V}(A)$ has n-comparison for some natural number $n$. Then $A$ has the Corona Factorization Property.

We proceed to study permanence properties of the strong CFP and of the CFP, in particular with respect to passing to ideals. Recall the definition of ideals (see below Corollary 2.5). If $S$ is a subset of $M$, then the set $I(S)$ of all elements $x \in M$, such that $x \leq k\left(y_{1}+y_{2}+\cdots+y_{r}\right)$ for some elements $y_{1}, y_{2}, \ldots, y_{r}$ in $S$ and some natural number $k$, is an ideal in $M$. We refer to $I(S)$ as the ideal generated by $S$. An ideal generated by a countable subset $S$ of $M$ is said to be countably generated. If $I=I(S)$ is generated by the countable set $S=\left\{y_{1}, y_{2}, y_{3}, \ldots\right\}$, then it is also generated by the set $\left\{y_{1}, y_{1}+y_{2}, y_{1}+y_{2}+y_{3}, \ldots\right\}$, and so every countably generated ideal is generated by an increasing sequence in $M$. Clearly, all ideals in a countable monoid are countably generated.

As usual, $I \triangleleft M$ is short for saying that $I$ is an ideal in $M$.

Every ideal in a conical refinement monoid is itself a conical refinement monoid.

Proposition 5.10. Let $M$ be a conical monoid and let $I$ be an ideal in $M$.

(i) If $M$ has the strong CFP, then so does the quotient monoid $M / I$.

(ii) If $M$ has the CFP and if I is countably generated, then $M / I$ has the CFP.

Proof. Let $x \mapsto \bar{x}$ denote the quotient mapping $M \rightarrow M / I$. For $x, y \in M$ one has $\bar{x} \leq \bar{y}$ in $M / I$ if and only if there exists $z \in I$ such that $x \leq y+z$ in $M$.

(i). Suppose that $M$ has the strong CFP. Let $x, y_{1}, y_{2}, \ldots$ be elements in $M$ and let $m$ be a natural number such that $\bar{x} \leq m \bar{y}_{n}$ for all $n$. Then $x \leq m y_{n}+z_{n}$ for some $z_{n} \in I$. Hence $x \leq m\left(y_{n}+z_{n}\right)$ for all $n$, whence $x \leq\left(y_{1}+z_{1}\right)+\left(y_{2}+z_{2}\right)+\cdots+\left(y_{k}+z_{k}\right)$ for some $k$ (because $M$ has the strong CFP). This shows that $\bar{x} \leq \bar{y}_{1}+\bar{y}_{2}+\cdots+\bar{y}_{k}$, and we conclude that $M / I$ has the strong CFP.

(ii). Suppose now that $M$ has the CFP. Let us first remark that each full sequence in $M / I$ lifts to a full sequence in $M$. Indeed, let $\left\{x_{n}\right\}$ be a sequence in $M$ such that $\left\{\bar{x}_{n}\right\}$ is full in $M / I$. Then $x_{n} \leq x_{n+1}+z_{n+1}$ for some $z_{n+1} \in I$. Accordingly, if we set $x_{n}^{\prime}=x_{n}+\left(z_{1}+z_{2}+\cdots+z_{n}\right)$, then $\overline{x_{n}^{\prime}}=\bar{x}_{n}$ and $x_{1}^{\prime} \leq x_{2}^{\prime} \leq x_{3}^{\prime} \leq \cdots$ in $M$. Let $\left\{v_{n}\right\}$ be an increasing sequence which generates the ideal $I$, and put $x_{n}^{\prime \prime}=x_{n}^{\prime}+v_{n}$. Then $\overline{x_{n}^{\prime \prime}}=\bar{x}_{n}$ and $\left\{x_{n}^{\prime \prime}\right\}$ is a full sequence in $M$. To see the latter, note first that $\overline{x_{n}^{\prime \prime}}$ is increasing by construction. Let $y \in M$ be given. Then $\bar{y} \leq m_{1} \bar{x}_{n_{1}}$ for some natural numbers $n_{1}$ and $m_{1}$. It follows that $y \leq m_{1} x_{n_{1}}^{\prime}+w$ for some $w$ in $I$. Next, $w \leq m_{2} v_{n_{2}}$ for some natural numbers $m_{2}$ and $n_{2}$. Put $m=\max \left\{m_{1}, m_{2}\right\}$ and $n=\max \left\{n_{1}, n_{2}\right\}$. Then

$$
y \leq m_{1} x_{n_{1}}^{\prime}+w \leq m_{1} x_{n_{1}}^{\prime}+m_{2} v_{n_{2}} \leq m x_{n}^{\prime}+m v_{n}=m x_{n}^{\prime \prime} .
$$

This shows that $\left\{x_{n}^{\prime \prime}\right\}$ is full in $M$.

Suppose that we are given a full sequence in $M / I$. By the argument above we may write this full sequence as $\left\{\bar{x}_{n}\right\}$, where $\left\{x_{n}\right\}$ is a full sequence in $M$. Let 
$\bar{y}_{1}, \bar{y}_{2}, \bar{y}_{3}, \ldots$ be another sequence in $M / I$ and let $m$ be a natural number such that $\bar{x}_{n} \leq m \bar{y}_{n}$ for all $n$. Then $x_{n} \leq m y_{n}+z_{n} \leq m\left(y_{n}+z_{n}\right)$ for some $z_{n}$ in $I$. As $M$ has the CFP we conclude that $x_{1} \leq\left(y_{1}+z_{1}\right)+\left(y_{2}+z_{2}\right)+\cdots+\left(y_{k}+z_{k}\right)$ for some natural number $k$. It follows that $\bar{x}_{1} \leq \bar{y}_{1}+\bar{y}_{2}+\cdots+\bar{y}_{k}$. This shows that $M / I$ has the CFP.

One can deduce from this proposition that the quotient of any separable $\mathrm{C}^{*}$ algebra of real rank zero with the CFP again has the CFP. This, however, is well known; cf. [10.

The CFP does not pass to ideals. Indeed, if $M$ is any conical monoid, then we can consider the monoid $M^{\sharp}:=M \sqcup\{\infty\}$, where addition is given by $u+\infty=\infty$ for all $u$ in $M^{\sharp}$. Then $M^{\sharp}$ has the CFP. Indeed, let $\left\{x_{n}\right\}$ and $\left\{y_{n}\right\}$ be sequences in $M^{\sharp}$ such that $\left\{x_{n}\right\}$ is full and $x_{n} \leq m y_{n}$ for some $m$. Then $x_{k}=y_{k}=\infty$ for all sufficiently large $k$, whence $x_{1} \leq y_{1}+y_{2}+\cdots+y_{k}$ if $k$ is chosen large enough so that $y_{k}=\infty$. However, $M$ is an ideal in $M^{\sharp}$, and being an arbitrary conical monoid, $M$ need not have the CFP (see, e.g., Proposition 5.17 below).

The situation is different for the strong CFP. We omit the trivial proof of the proposition below.

Proposition 5.11. If $M$ is a conical monoid with the strong Corona Factorization Property, then every ideal in $M$ also has the strong Corona Factorization Property.

In the case of conical refinement monoids the strong CFP and the CFP are related as follows:

Proposition 5.12. Let $M$ be a conical refinement monoid. Then $M$ has the strong Corona Factorization Property if and only if every ideal in $M$ has the Corona Factorization Property.

Proof. If $M$ has the strong CFP, then so does every ideal $I$ in $M$, cf. Proposition 5.11, and if $I$ has the strong CFP, then $I$ also has the CFP. (Note that in this direction of the proof we have not used that $M$ is a refinement monoid.)

To prove the reverse direction we need to establish the following fact. Suppose that $x, y \in M$ and $m \in \mathbb{N}$ are such that $x \leq m y$. Then there is $y^{\prime}$ in $M$ such that $x \leq m y^{\prime}, y^{\prime} \leq x$, and $y^{\prime} \leq y$. Indeed, $m y=x+t$ for some $t$ in $M$. Hence, by [18, Lemma 1.9], we have a refinement

\begin{tabular}{c|cccc} 
& $y$ & $y$ & $\cdots$ & $y$ \\
\hline$x$ & $a_{1}$ & $a_{2}$ & $\cdots$ & $a_{m}$ \\
$t$ & $b_{1}$ & $b_{2}$ & $\cdots$ & $b_{m}$
\end{tabular}

with $a_{1} \leq a_{2} \leq \cdots \leq a_{m}$ (and $b_{1} \geq b_{2} \geq \cdots \geq b_{m}$ ). Put $y^{\prime}=a_{m}$. Then $y^{\prime} \leq x$, $y^{\prime} \leq y$ and $m y^{\prime} \geq a_{1}+a_{2}+\cdots+a_{m}=x$.

Now assume that every ideal in $M$ has the CFP (actually we need only assume that every singly generated ideal in $M$ has the CFP). We wish to show that $M$ has the strong CFP. Let $x, y_{1}, y_{2}, \ldots$ in $M$ and $m \in \mathbb{N}$ be given such that $x \leq m y_{n}$ for every $n$. Let $I$ be the ideal in $M$ generated by $x$. Then $x$ is an order unit in $I$, so the constant sequence $\left\{x_{n}\right\}$, with $x_{n}=x$ for all $n$, is a full sequence in $I$. Use the fact established above to find $y_{n}^{\prime}$ in $M$ such that $y_{n}^{\prime} \leq x, y_{n}^{\prime} \leq y_{n}$, and $x \leq m y_{n}^{\prime}$ for all $n$. Then each $y_{n}^{\prime}$ belongs to $I$, and $x_{n}=x \leq m y_{n}^{\prime}$ for all $n$. Since $I$ is assumed 
to have the CFP there is a natural number $k$ such that

$$
x=x_{1} \leq y_{1}^{\prime}+y_{2}^{\prime}+\cdots+y_{k}^{\prime} \leq y_{1}+y_{2}+\cdots+y_{k} .
$$

This proves that $M$ has the strong CFP.

We can now characterize the class of $\mathrm{C}^{*}$-algebras $A$ (of real rank zero) for which the monoid $\mathrm{V}(A)$ has the strong CFP:

Theorem 5.13. Let $A$ be a $\sigma$-unital $C^{*}$-algebra of real rank zero. Then $\mathrm{V}(A)$ has the strong Corona Factorization Property (for monoids) if and only if every ideal I in $A$ has the Corona Factorization Property (for $C^{*}$-algebras).

Proof. The assumptions on $A$ imply that $\mathrm{V}(A)$ is a conical refinement monoid. The mapping $I \mapsto \mathrm{V}(I) \subseteq \mathrm{V}(A)$ from ideals $I$ in $A$ into subsets of $\mathrm{V}(A)$ gives a bijection between ideals in $A$ and ideals in $\mathrm{V}(I)$ (in the case where $A$ is of real rank zero). Each ideal in $A$ is separable and of real rank zero. Hence, by Theorem 5.8, the condition that every ideal $I$ in $A$ has the $\mathrm{CFP}$ (for $\mathrm{C}^{*}$-algebras) is equivalent to the condition that every ideal in $\mathrm{V}(A)$ has the CFP (for monoids). The theorem therefore follows from Proposition 5.12 above.

We end this section by discussing an important consequence of having the CFP:

Theorem 5.14. Let $M$ be a conical refinement monoid, and let $u$ be an element in $M$ such that $m u$ is properly infinite for some natural number $m$. Then $u$ itself is properly infinite if one of the two conditions below holds:

(i) $M$ has the strong CFP,

(ii) $M$ has the CFP and $u$ is an order unit in $M$.

Proof. Upon replacing $M$ by the ideal generated by $u$ (and using Proposition 5.12) it suffices to consider case (ii). By Corollary 4.4 there is a sequence $t_{1}, t_{2}, t_{3}, \ldots$ of order units in $M$ such that $t_{1}+t_{2}+\cdots+t_{k} \leq u$ for all $k$, and $m t_{n}$ is properly infinite for all $n$. It follows in particular that $u \leq m t_{n}$ for all $n$. Now, because $M$ has the CFP, there exists $k$ such that $u \leq t_{1}+t_{2}+\cdots+t_{k}$. Applying the CFP again we obtain that $u \leq t_{k+1}+t_{k+2}+\cdots+t_{l}$ for some $l>k$. This shows that

$$
2 u \leq t_{1}+t_{2}+\cdots+t_{k}+t_{k+1}+t_{k+2}+\cdots+t_{l} \leq u,
$$

whence $u$ is properly infinite.

Corollary 5.15. Let $A$ be a $\sigma$-unital $C^{*}$-algebra of real rank zero, and let $p$ be a projection in $A$ such that the $m$-fold direct sum $p \oplus p \oplus \cdots \oplus p$ is properly infinite (in $M_{m}(A)$ ) for some natural number $m$. Then $p$ itself is properly infinite if one of the following two conditions below holds:

(i) Every ideal in $A$ has the CFP,

(ii) A has the CFP and $p$ is a full projection in $A$.

Proof. This follows from Theorem 5.14 above together with Theorems 5.8 and 5.13 . Also use that if $p$ is a projection in $A$, then $p$ is properly infinite (as a projection in $A$ ) if and only if $[p]$ is properly infinite (as an element in $\mathrm{V}(A)$ ).

It follows from this result that $\mathrm{C}^{*}$-algebras with the $\mathrm{CFP}$ satisfy the following dichotomy: either all full projections are properly infinite or no full projections are properly infinite.

The corollary below was proved in an unpublished paper by S. Zhang. (One can drop the separability assumption by passing to a suitable separable sub-C*-algebra.) 
Corollary 5.16. A separable simple $C^{*}$-algebra of real rank zero with the Corona Factorization Property is either stably finite or purely infinite.

Proof. Suppose that $A$ is a separable simple $\mathrm{C}^{*}$-algebra of real rank zero and with the CFP. Then the monoid $\mathrm{V}(A)$ is a simple, countable refinement monoid with the CFP (by Theorem 5.8). If all elements in $\mathrm{V}(A)$ are finite, then all projections in $A$ and in matrix algebras over $A$ are finite, whence $A$ is stably finite. Suppose that $\mathrm{V}(A)$ contains an infinite element. Then $\mathrm{V}(A)$ contains a properly infinite element $u$ (because all infinite elements in a simple monoid are properly infinite). Let $v$ be any other non-zero element in $\mathrm{V}(A)$. Then $v$ is an order unit (because $\mathrm{V}(A)$ is simple), and so $u \leq m v$ for some natural number $m$. It follows that $m v$ is properly infinite (because $m v+m v \leq u \leq m v$, the former inequality holds because $u$ is a properly infinite order unit). By Theorem 5.14 we get that $v$ is itself properly infinite. This shows that all non-zero elements of $\mathrm{V}(A)$ are properly infinite. This translates into the statement that all non-zero projections in $A$ (and in matrix algebras over $A$ ) are properly infinite. As $A$ is assumed to be of real rank zero, this implies that $A$ is purely infinite.

It is an open problem if all $\mathrm{C}^{*}$-algebras of real rank zero have the CFP. At the level of monoids, the analog problem has a negative answer.

Proposition 5.17. There exists a simple conical refinement monoid $M$ with an order unit $u$ such that $u$ is finite while $2 u$ is properly infinite. In particular, $M$ does not have the CFP.

Proof. The monoid $M_{0}=\{0, u, \infty\}$, where $u+u=\infty$, is simple and conical. The element $u$ is an order unit in $M_{0}, u$ is finite, while $2 u=\infty$ is infinite. By [19], $M_{0}$ is a unitary submonoid of a simple conical refinement monoid $M$. Obviously $2 u$ remains infinite in $M$. Since $M_{0}$ is cofinite in $M$, we see that $u$ is an order unit in $M$. If $u+x=u$ for some $x \in M$, then $x \in M_{0}$ because $M_{0} \subseteq M$ is unitary, whence $x=0$. Therefore $u$ remains finite in $M$.

The last conclusion follows from Theorem 5.14

The monoid $M_{0}$ from the proof of Proposition 5.17 above has some interesting features. It is a simple monoid with a finite element $u$ such that $2 u$ is properly infinite. At the same time it does satisfy the strong CFP. (This is easy to check; there are not so many ways in which one can choose the elements $x, y_{1}, y_{2}, \ldots$ ) This shows that in Theorem 5.14 one cannot omit the assumption that the monoid has the refinement property.

\section{ACKNOWLEDGEMENTS}

The first and second named authors were partially supported by a MEC-DGESIC grant (Spain) through Project MTM2008-0621-C02-01/MTM, and by the Comissionat per Universitats i Recerca de la Generalitat de Catalunya. The third named author was supported by a grant from the Danish Natural Science Research Council (FNU). The second named author wishes to thank G. del Peral for inspiring conversations concerning the results in Section 3. Part of this research was carried out during visits of the first and third named authors to UAB (Barcelona), of the second named author to SDU (Odense), and of the two first mentioned authors to Copenhagen. We wish to thank all parties involved for the hospitality extended to us. 


\section{REFERENCES}

[1] P. Ara and E. Pardo, Refinement monoids with weak comparability and applications to regular rings and $C^{*}$-algebras. Proc. Amer. Math. Soc. 124 (1996), 715-720. MR.1301484 (96f:46124)

[2] P. Ara, K. R. Goodearl, K. C. O'Meara, and E. Pardo, Separative cancellation for projective modules over exchange rings. Israel J. Math. 105 (1998), 105-137. MR1639739 (99g:16006)

[3] G. Aranda, K.R. Goodearl, F. Perera, and M. Siles Molina, Non-simple Purely Infinite Rings. Amer. J. Math. 132 (2010), no. 3, 563-610. MR2666902

[4] L.G. Brown, Stable isomorphism of hereditary subalgebras of $\mathrm{C}^{*}$-algebras. Pacific J. Math. 71 (1977), 335-348. MR0454645 (56:12894)

[5] L. G. Brown and G. K. Pedersen, C*-algebras of real rank zero. J. Funct. Anal. 99 (1991), 131-149. MR1120918 (92m:46086)

[6] H. Dobbertin, Refinement monoids, Vaught monoids, and Boolean algebras. Math. Ann. 265 (1983), 473-487. MR721882 (85e:06016)

[7] G. A. Elliott and D. Kucerovsky, An abstract Voiculescu-Brown-Douglas-Fillmore absorption theorem. Pacific J. Math. 198 (2001), 385-409. MR1835515 (2002i:46052)

[8] I. Hirshberg, M. Rørdam, and W. Winter, $C_{0}(X)$-algebras, stability and strongly selfabsorbing C*-algebras. Math. Ann. 339 (2007), 695-732. MR2336064 (2008j:46040)

[9] J. Hjelmborg and M. Rørdam, On stability of C*-algebras. J. Funct. Anal. 155 (1998), 153171. MR $1623142(99 \mathrm{~g}: 46079)$

[10] D. Kucerovsky and P. W. Ng, S-regularity and the corona factorization property. Math. Scand. 99 (2006), 204-216. MR2289022 (2009g:46103)

[11] E. Ortega, F. Perera and M. Rørdam, The Corona Factorization Property, Stability, and the Cuntz semigroup of a $C^{*}$-algebra. International Mathematics Research Notices (2011); doi: 10.1093/imrn/rnr013

[12] E. Pardo, Metric completions of ordered groups and $K_{0}$ of exchange rings. Trans. Amer. Math. Soc. 350 (1998), 913-933. MR1376552 (98e:46088)

[13] F. Perera, The structure of positive elements for $\mathrm{C}^{*}$-algebras with real rank zero. Int. J. Math. 8 (1997), 383-405. MR1454480 (98i:46058)

[14] F. Perera and M. Rørdam, AF-embeddings into $\mathrm{C}^{*}$-algebras of real rank zero. J. Funct. Anal. 217 (2004), 142-170. MR2097610 (2005g:46109)

[15] M. Pimsner, S. Popa, and D. Voiculescu, Homogeneous $\mathrm{C}^{*}$-extensions of $C(X) \otimes K(H)$, I. J. Operator Theory 1 (1979), 55-108. MR526291(82e:46093a)

[16] M. Rørdam, Stability of C*-algebras is not a stable property. Doc. Math. 2 (1997), 375-386. MR.1490456 (98i:46060)

[17] M. Rørdam, A simple $\mathrm{C}^{*}$-algebra with a finite and an infinite projection. Acta Math. 191 (2003), 109-142. MR2020420 (2005m:46096)

[18] F. Wehrung, Injective positively ordered monoids, I. J. Pure Appl. Algebra 83 (1992), 43-82. MR.1190444 (93k:06023)

[19] F. Wehrung, Embedding simple commutative monoids into simple refinement monoids. Semigroup Forum 56 (1998), 104-129. MR1490558 (99b:20092)

[20] S. Zhang, Matricial structure and homotopy type of simple $\mathrm{C}^{*}$-algebras with real rank zero. J.Operator Theory 26 (1991), 283-312. MR1225518 (94f:46075)

Department of Mathematical Sciences, Norwegian University of Science and TechNOLOGY, NO-7491 TRONDHEIM, NORWAY

E-mail address: Eduardo.Ortega@math.ntnu.no

Departament de Matemàtiques, Universitat Autònoma de Barcelona, 08193 BellaTERra, Barcelona, SPAin

E-mail address: perera@mat.uab.cat

Department of Mathematical Sciences, University of Copenhagen, UniversitetsParken 5, DK-2100, Copenhagen $\varnothing$, Denmark

E-mail address: rordam@math.ku.dk 